\title{
Capturing the Complexity of Cognitive Computing Systems: Co-Adaptation Theory for Individuals
}

\author{
Rasha Alahmad \\ University of Michigan \\ School of Information \\ Ann Arbor, MI 48109, USA \\ rashama@umich.edu
}

\author{
Lionel P. Robert Jr. \\ University of Michigan \\ School of Information \\ Ann Arbor, MI 48109, USA \\ lprobert@umich.edu
}

\begin{abstract}
Cognitive computing systems (CCS) are the new generation of automated IT systems that mimic human cognitive capabilities. CCS reshape the interaction between humans and machines and challenge our traditional assumptions of technology use and adoption. This work introduces co-adaptation and defines it as the series of activities that a user and a system engage in simultaneously to make the system fit the user. Co-adaptation involves two types of adaptation: human adaptation and machine adaptation. Human adaptation refers to the user either changing their behavior to adjust to the technology or changing the technology to adjust to their use. Machine adaptation refers to the system adapting itself to fit users' needs. We use polynomial regression and response surface analysis to examine the impact of co-adaptation on individual performance. We add to previous work by offering a solid theoretical argument with supporting evidence that congruence between human adaptation and machine adaptation plays a critical role in determining the impact of technology use on individuals and their performance.
\end{abstract}

\section{CCS CONCEPTS}

- Information systems $\rightarrow$ Information Systems Applications $\rightarrow$ Computing Platforms

KEYWORDS: Technology Adaptation; Co-adaptation; Cognitive Computing; Artificial Intelligence; IT identity

\section{ACM Reference format:}

Rasha Alahmad and Lionel P. Robert Jr. 2021. Capturing the Complexity of Cognitive Computing Systems: Co-Adaptation Theory for Individuals. In Proceedings of 2021 ACM SIGMIS-CPR '21, fune 30, 2021, Virtual Event, Germany, ACM, New York, NY, USA, 2 pages. https://doi.org/10.1145/3458026.3462148

\section{Introduction}

Cognitive computing is a broad term that refers to self-learning systems that use data mining techniques, pattern recognition,

\footnotetext{
Permission to make digital or hard copies of part or all of this work for personal or classroom use is granted without fee provided that copies are not made or distributed for profit or commercial advantage and that copies bear this notice and the full citation on the first page. Copyrights for third-party components of this work must be honored. For all other uses, contact the Owner/Author(s).

SIGMIS-CPR '21, June 30, 2021, Virtual Event, Germany.

(C) 2021 Copyright is held by the owner/author(s).

ACM ISBN 978-1-4503-8406-3/21/06. https://doi.org/10.1145/3458026.3462148
}

and natural language processing to mimic the human mind's cognitive capabilities. Cognitive computing is the nextgeneration application of AI-based solutions that enable humanmachine interactions similar to interpersonal interactions. The power of teaming up with humans for performing tasks redefines the nature of the relationship between humans and machines [17]. Human-machine interaction boundaries are elastic and constantly adjusting, which requires us to rethink the symbiotic relationship and the direct implications on individuals and their performance. There is a lack of theoretical discussion and an integrated body of literature on cognitive computing's impact on organizations and individuals [2].

One of the distinguishing characteristics of CCS is adaptability. As described by the Cognitive Computing Consortium [3], CCS are adaptable and interactive systems that can learn from their interactions with individuals. Unlike previous technologies, CCS can adapt itself to the user as the user adapts to it. This coadaptation is both new and vital to understanding users' interactions with CCS. Co-adaptation occurs when both the user and the technology adapt to each other simultaneously to make the technology better fit the user. Co-adaptation poses a new theoretical challenge to the current notion of adaptation. For example, does it matter if the user adapts more to the system, or should the system adapt more to the user? Answering these questions should help to understand CCS use and its important implications for individual performance. Despite the importance of co-adaptation, the literature has lacked a theoretical view for understanding how it occurs and what impact co-adaptation has on users.

The emerging complex relationship between humans and machines $[4,5,18]$ changes the typical instrumental supporting role of technology [6] into a collaborative and algorithmic role. As technology has evolved from supporting individuals in performing tasks towards becoming more involved in the activities, it has impacted individuals and their performance. In the era of human-machine symbiosis, it becomes hard to draw lines between individuals' performativity and the performativity of machines due to the increased embeddedness of technology in the nature of work [7]. Co-adaptation has the potential to reshape users' personal identity. Bidirectional relationships propel individuals to adjust themselves as the machines do to keep maintaining a constant interaction. However, it is simply not clear if the interaction with the machine will lead to an expanded self or if it will not impact the self at all. It is not clear 
if co-adaptation will motivate users to tap into machine resources to enhance their potential efficacy or consider CC systems merely as machines to help them achieve specific goals. IT identity theory should be vital to helping us understand the effect of co-adaptation on personal identity. IT identity suggests that technology embeddedness into individuals' lives leads to viewing the use of technology as integral to their sense of self [8]. Thus, the current study will help understand the impact of co-adaptation on individuals' identity and performance.

\section{Theoretical Background}

CCS are the new generation of automated Information Technology systems that mimic human cognitive capabilities. CCS reshape the interaction between humans and machines and challenge our traditional assumptions of IT systems. The systems in the IS field are still seen as tools bundled with functionalities to help users reach specific outcomes [1,9]. For instance, tasktechnology fit, Adaptive Structuration Theory, Fit Appropriation Model, Coping Model of User Adaptation, Adaptive Structuration Theory for Individuals are critical theories in IS literature that considers IT systems as tools used by individuals to achieve desirable outcomes. However, CCS is an adaptive and interactive system capable of engaging with humans in bilateral relationships [1]. CCS fundamentally differs from previous IT systems, which question the applicability of existing technology use and adaptation knowledge. Our study extends the research on technology adaptation by introducing the co-adaptation framework to the IS community.

Technology adaptation is defined as the cognitive and behavioral efforts exerted by individuals to manage perceived consequences of technology implementation in their work environment [10]. Adaptation is a key concept to understand change and adjustment following a new technology deployment. Research has shown that only through experimenting with technology individuals discover its ramifications [11]. Individuals frequently modify and fundamentally change technology features to accomplish their tasks. It is important to understand adaptation behavior because technology rarely fits with existing tasks and routines [12], and achieving task efficiency with technology largely depends on technology modification [13].

We define co-adaptation as the series of activities that a user and a system engage in simultaneously to make the system fit the user. Co-adaptation involves two types of adaptation: human adaptation and machine adaptation. Human adaptation refers to the user either changing their behavior to adjust to the technology or changing the technology to adjust to their use. Machine adaptation refers to the system adapting itself to fit users' needs. We propose co-adaptation as a logical grouping of factors each factor impacts distinctly on the individuals' behavior and performance. Prior studies have solely considered human adaptation $[14,15,16]$; however, our research goes a step further by considering machine adaptation. Machine adaptation is an additive adaptation perspective that helps to understand human-machine interaction. While these two aspects of adaptation are conceptually different, they are not entirely independent. Co-adaptation aspects coexist and are correlated because the change in human adaptation is associated with machine adaptation change.

\section{Method}

There was a total of 46 Amazon Alexa users who participated in the survey. The analysis was conducted using the polynomial regression model and Response surface analysis. Polynomial regression procedures require two steps: first, entering $\mathrm{X}, \mathrm{Y}$ values to test the linear relationship (Equation 1); second, entering higher-order values $\left(X^{2}, Y^{2}\right)$ and the product value (XY) to test the curvilinear relationship. Our preliminary findings indicate a congruence effect (Figure 1). Thus, as the degree of equality between the two aspects of co-adaptation increases, the identification with the system becomes stronger at a faster rate.

$$
Z=b_{0}+b_{1} X+b_{2} Y+b_{3} X^{2}+b_{4} X Y+b_{5} Y^{2}+e
$$

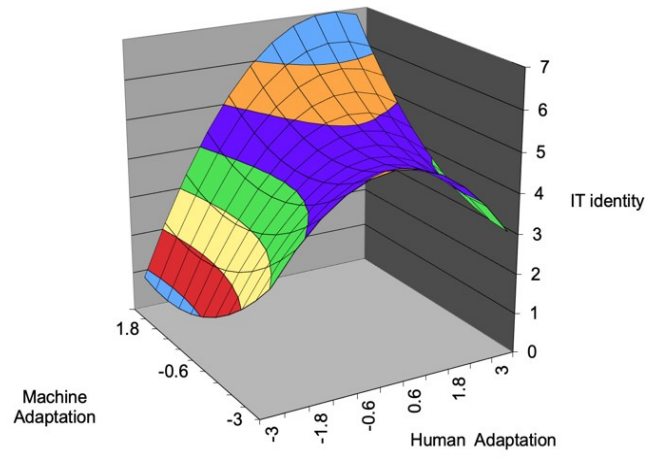

Figure 1: Response Surface Analysis for Co-adaptation Predicting IT identity

\section{Contributions}

First, our work contributes to adaptation literature by introducing the concept of co-adaptation as a new and pivotal theoretical view of adaptation. Prior studies have viewed adaptation from the perspective of humans adapting the technology or adapting to the technology. If we had taken that approach, we would not be able to fully understand the role the CCS has on technology use. Our findings show that machine adaptation is an additional key aspect of adaptation and that the concept of co-adaptation matters in understanding CCS adaptivity and its impact on individual performance. Second, we add to previous work on adaptation by offering a solid theoretical argument with supporting evidence that congruence between human adaptation and machine adaptation plays a critical role in determining the impact of system use and adaptation on individual performance. Our findings show that congruence between human adaptation and machine adaptation increases identification with the system. 


\section{REFERENCES}

[1] Sebastian Schuetz and Viswanath Venkatesh, 2020. The Rise of Human Machines: How Cognitive Computing Systems Challenge Assumptions of User-System Interaction. Journal of the Association for Information Systems, 21, no. $2,460-482$

[2] Hemant Jain, Balaji Padmanabhan, Paul A. Pavlou, and Raghu T. Santanam, eds, 2018. Call for papers-Special Issue of Information Systems ResearchHumans, Algorithms, and Augmented Intelligence: The Future of Work, Organizations, and Society. Information Systems Research, 29, no. 1, 250-251.

[3] Cognitive Computing Consortium, 2014. Cognitive Computing Systems, last modified December 15, 2020, https://cognitivecomputingconsortium.com

[4] Hind Benbya, Ning Nan, Hüseyin Tanriverdi, and Youngjin Yoo, 2020. Complexity and Information Systems Research in the Emerging Digital World. MIS Quarterly, 44, no. 1, 1-17.

[5] Arun Rai, Panos Constantinides, and Saonee Sarker, 2019. Editor's Comments: Next-Generation Digital Platforms: Toward Human-AI Hybrids. MIS Quarterly, 43, no. 1, iii-x.

[6] Kai Riemer and Sandra Peter, 2020. The Robo-apocalypse plays out in the quality, not in the quantity of work. Journal of Information Technology, 35, no. 4, 310-315.

[7] Mary Beth Watson-Manheim and Stefan Klein, 2019. Conceptualizing Hidden Human Work in a Technology Intensive Work Environment. In OCIS Shanghai Conference at Harvard.

[8] Michelle Carter and Varun Grover, 2015. Me, My Self, And I (T). MIS Quarterly, 39, no. 4, 931-958.

[9] Izak Benbasat and Robert W. Zmud, 2003. The Identity Crisis Within the IS Discipline: Defining and Communicating the Discipline's Core Properties. MIS Quarterly, 27, no. 2, 183-194
[10] Anne Beaudry and Alain Pinsonneault, 2005. Understanding User Responses to Information Technology: A Coping Model of User Adaptation. MIS Quarterly, 29, no. 3, 493-524.

[11] Marcie J. Tyre and Wanda J. Orlikowski, 1994. Windows of Opportunity: Temporal Patterns of Technological Adaptation in Organizations. Organization Science, 5 , no. 1, 98-118.

[12] Dorothy A. Leonard, 1988. Implementation as Mutual Adaptation of Technology and Organization. Research Policy, 251-267.

[13] John M. Dutton and Annie Thomas, 1984. Treating Progress Functions as a Managerial Opportunity. Academy of Management Review, 9, no. 2, 235-247.

[14] Henri Barki, Ryad Titah, and Céline Boffo, 2007. Information System UseRelated Activity: An Expanded Behavioral Conceptualization of Individuallevel Information System Use. Information Systems Research, 18, no. 2, 173 192.

[15] Marie-Claude Boudreau and Daniel Robey, 2005. Enacting Integrated Information Technology: A Human Agency Perspective. Organization Science, 16 , no. 1, 3-18.

[16] Kurt W. Schmitz, James TC Teng, and Kimberly J. Webb, 2016. Capturing the Complexity of Malleable IT Use: Adaptive Structuration Theory for Individuals. MIS Quarterly, 40, no. 3, 663-686.

[17] Lionel Robert, Rasha Alahmad, Sangmi Kim, Connor Esterwood, Sangseok You, and Qiaoning Zhang, 2020. A Review of Personality in Human Robot Interactions, Foundations \& Trends in Information Systems, (pdf), 4(2), 107 210. http://dx.doi.org/10.1561/2900000018

[18] Lionel Robert, Casey Pierce, Liz Marquis, Sangmi Kim, and Rasha Alahmad, 2020. Designing Fair AI for Managing Employees in Organizations: A Review, Critique, and Design Agenda, Human-Computer Interaction, 35(5-6), 545-575. https://doi.org/10.1080/07370024.2020.1735391 